\title{
Strengthening Malaria Service Delivery through Measurement of Quality Improvement of Performance Standards in Nigeria: evaluation of Community Health Workers Performance
}

\author{
Article by Bright Orji ${ }^{1}$, William Brieger ${ }^{2}$, Lindiwe Innocentia ${ }^{3}$ \\ 1,3 Texila American University \\ ${ }^{2}$ Public Health, the Johns Hopkins Bloomberg \\ E-mail:bright2orji@yahoo.com ${ }^{1}$
}

\begin{abstract}
This study monitored quality improvement performance standards of Community Health Workers $(C H W s)$ who were providing integrated community case management of malaria, pneumonia and diarrhea (iCCM) in two selected LGAs. One hundred and fifty-two trained CHWs providing iCCM services in Akwa Ibom State, Nigeria were monitored and assessed using one page tool on quality improvement performance standards. The tool has 37 performance criteria (PC) to measure CHWs' knowledge, skills and competence in 3 sections: History taking and Examination; Conducting RDTs for Malaria; and Illness Management. Trained assessors observed CHWs providing services. Each correctly performed criterion was scored 1 point. Four rounds of assessments were conducted at an interval of two months from June 2011 - March, 2012 as part of the efforts to strengthen services provided by CHWs. The result shows that during Round 1 CHWs achieved an average of $19(52.2 \%)$ performance standards. This rose to 25 (67.5\%) in Round 2; 28 $(75.6 \%)$ in 3 and $30(81.1 \%)$ in round 4 and $(p=0.00)$. Therefore, the number of $C H W$ s that scored $>=80$ increased from 79 in round-1 to 124 in round-4. PC that needed to be strengthened the most included reinforcement on checking RDT expiry date, recording results, and safe disposing of sharps. Providing feedback in-between rounds of assessments contributed to CHWs improved performance during the subsequent round. The feedback mechanism provides the platform for addressing the barriers that limits the CHWs extent of achieving performance standards for malaria improved service delivery
\end{abstract}

Keywords: Measurement, Quality improvement performance standards, community health workers, integrated community case management of malaria, pneumonia and diarrhea.

\section{Introduction}

Globally, the number of malaria cases has been in the decline. In 2016, the number of malaria cases declined to 216 million from 237 million cases in 2010 (WMR, 2017). Estimated incidence of malaria cases dropped from 63 cases to 76 cases per 1000 per population at risk of the disease between 2010 and 2016. Similarly, global malaria deaths dropped between the same periods. Estimated annual malaria deaths in 2016 was 445,000. In Nigeria, percent of persons with suspected malaria receiving a diagnostic test (RDT and/or microscopy) increased from $17 \%$ in 2012 to $39 \%$ in 2016. In 2015, $41 \%$ of children under-five years of age that reported fever, only $13 \%$ were tested using RDTs while $38 \%$ of them received ACTs the recommended medicine for malaria treatment (MIC, 2015).

The increase in number of people receiving malaria care in Nigeria has over-stretched the health system; resulting in poor availability of infrastructures, equipment, supplies and heavy workload for the few standing health workers. These may have compromised quality of care provided by the available service providers (Adrienne Kols, Young-Mi Kim, Eva Bazant, Edgar Necochea, Joseph Banda and Stacie Stender, 2015). One study in Nigeria reported quality of care in a hospital to be $38.8 \%$ and described this as suboptimal and totally unacceptable (Ariba AJ., Thanni LO., Adebayo EO; 2007) while another study showed increase in non-adherence to treatment protocol among cases of maternal death in 2002-2004 compared with 1999-2001 though this was not statistically significant but enough concern for quality of 
DOI: $10.21522 / \mathrm{TIJPH} .2013 .06 .02 . A r t 010$

ISSN: $2520-3134$

care. One of the reasons for poor quality of care was the shortage of human resource for health. Therefore, engagement of CHWs as part of the efforts in the implementation of task shifting policy to address the challenges of poor availability of human resource for health becomes inevitable. Various studies have shown that trained CHWs can use RDTs as a component of home management of malaria but only few studies have monitored and assessed quality of services provided by the CHWs.

This has prompted focus on CHWs performance assessment using evidence-based standards for maternal, newborn and child health care. The standards are developed from evidence-based practices and includes practical perspective on technical areas as well as emphases clients' centered care. Clients' centered care are services that recognizes and respects patients' rights and human dignity including cultural orientations (Necochea E., Tripathi V., MI-Kim Y., Akran N., Hyjazi Y., Luz vaz M., Otolorin EO., Pleah T., Rashidi T., Bishanga D., 2015). Implementation of evidence interventions and client's centered care to reduce maternal death in Nigeria has been very tasking. Some of the reasons given for this include poor documentation, and gaps in the measurement of quality of care that might have hindered the delivery of quality care by the service providers (Kabo I., Otolorin EO., Williams E., et al, 2016). In one study, Kinney et al opine that if quality of care cannot be measured, identification of quality gaps will be challenged and therefore affect the ability to reduce the gaps. On the other hand, poor documentation will lead to poor quality data that is critical for decision-making to address health challenges and improve malaria service delivery among countries on the pathway to malaria elimination.

There has been increasing national and international attention to the need to improve the quality of malaria services. In 2010 and 2011 World Health Organization adopted "counting out malaria" as a theme for the years' world malaria day; in an effort to draw attention to quality of malaria services and the need for strong health information, monitoring and evaluation system.

Kabo et al, 2016 has noted that maternal and newborn health outcomes in Nigeria can be improved through monitoring and measurement of quality improvement performance standards.

In Bauchi State, Nigeria (Kabo I., Otolorin EO., Williams E., et al, 2016) demonstrated that application of provider performance quality improvement assessment to maternal and newborn health services in 20102014 among 23 secondary health facilities resulted in well over $82 \%$ achievement of performance standards from baseline within four years of program implementation. While service providers compliance to quality improvement performance standard improved over time from 8\% (2010) baseline to $92 \%$ (2013) leading to the decline of maternal mortality rate from 4113/100,000 live births in 2010 to 704 in 2014.

There are only very few studies in Nigeria on the quality performance of community health workers. However, studies in other seven countries that shared similar characteristics with Nigeria such as Bangladesh, India, Nepal, Pakistan, the Philippines and Tanzania that involved CHWs have reported high impact and quality performance and contributions on diagnosis and treatment of pneumonia that reduced risk of death by $36 \%$ in children in addition to $24 \%$ overall risk of deaths in children living in those countries (Perry HB., Zulliger R., Rogers MM., 2014). These evidences have continued to support active involvement and participation of CHWs in public health interventions as part of the key strategy to reduce the effect of critical shortages of health workers in the developing countries.

This current effort monitored and measured the CHWs achievement of performance standards in providing quality management of febrile illnesses in two selected LGAs, in Nigeria. The study will answer the research question what is the effect of regular monitoring and measurement of quality improvement performance of CHWs providing integrated community case management of malaria, pneumonia and diarrhea? Study objective - The study will investigate the CHWs achievement of performance standards in the delivery of integrated community case management (iCCM) of malaria, diarrhea and simple respiratory infection in Akwa Ibom State, Nigeria. The project will specifically. Describe the effect of regular quality assessments on CHW's performance. 


\section{Method}

The study was measurement over time implementation research to monitor and strengthen quality improvement performance of the CHWs providing integrated community case management of malaria, pneumonia and diarrhea (iCCM) in Akwa Ibom State, Nigeria. Standards used in this study include taking history/examination; conducting RDTs and appropriate management of febrile illness.

152 CHWs selected by the communities were studied. The selection criteria used by the community included persons living in the community where they served, ability to read and write, agreed to volunteer services, if married must have support of spouse, willingness to attend monthly meetings and submit reports. In some other communities, criteria included previous experience especially community with onchocerciasis experience with active CHWs. Community meetings were held, and usually included men, women, youths and visitors residents in the community. The CHWs were mainly farmers, traders, retirees, civil servants etc.

Total of two thousand, two hundred and six clients were reached by the CHWs during the project implementation period June 2011 - March, 2012.

\section{Tool and data management}

The study used purposive sampling technique because all the $152 \mathrm{CHWs}$ were studied for quality improvement of performance standards in the delivery of iCCM.

The state ministry of health partnered with implementing agency and developed one page tool for quality performance standards - monitoring the quality of services provided by the CHWs in two selected LGAs of Akwa Ibom State, Nigeria. The tool has 37 performance criteria (PC) that measured CHW knowledge, skills and competence in 3 sections: History taking and Examination; Conducting RDTs for Malaria; and Illness Management. Each section of the performance criteria has between 11 to 13 verifiable criteria. The tool was used to observe the CHWss while providing services at the community; each correctly performed criterion was scored 1 point.

Four rounds of assessments were conducted after the baseline at an interval of two months between project implementation periods June 2011 - March, 2012. The results of the previous assessments was used to provide feedback to the CHWs before the next round of evaluation. The post-evaluation data collected during the iCCM project were re-analyzed using SPSS version 21.0 series for Windows.

In the analysis, descriptive statistics such as mean, mode, median, standard deviation percentage, as well as graphic illustrations were used in characterizing the data of the CHWs socio demographic characteristics. These characteristics include the CHWs' sex, age, education and vocations. Also, CHWs improvement in the delivery of quality services (defined as taking history/examination, conducting RDTs and appropriate management of febrile illness) was measured in percentages (\%). A score $\geq 80 \%$ was set as quality improved service delivery based on Jhpiego's participant's pass mark at trainings; while $\leq 80 \%$ is adjudged as non-quality improved services using the round-4 endline assessment.

The CHWs meet monthly to share challenges, success stories, replenish stock of medicines and get feedback on their performance. The supervising health worker used the opportunity to administer the supervisory checklist to monitor and assess the performance of the CHW based on their bi-monthly reports and provide support and feedback on areas that needed improvement.

The tool was administered quarterly in collaboration with the Local government staff who also provided supportive supervision to the CHWs; and three rounds of supervision visits were provided

\section{Results}

The sample size for the study was 152 CHWs. The 152 CHWs worked through the end of the project. There was no attrition, and performance of the CHWs were measured at the interval of two to three months. Of the 152 CHWs provided iCCM services during the period under review Table 1 shows the sociodemographic characteristics of the CHWs. 
DOI: $10.21522 / \mathrm{TIJPH} .2013 .06 .02 . A r t 010$

ISSN: $2520-3134$

One hundred and fifty-two (152) CHWs were studied. $21 \%$ were male while $79 \%$ were female CHWs. The CHWs overall mean age was 36.8 years with a standard deviation of \pm 8.7 years. The mean age for the male CHWs was 37.6 years with standard deviation of \pm 4.3 while the mean age for the female CHWs was 36.6 years with standard deviation of \pm 9.5 . The youngest age of the study CHWs was 30 years while the oldest age was 49 years old with a range of 30-49. The CHWs were assigned to the following age groups: $<36$ years, and $>36$ years. The mean age of the CHWs was used as the cut-off point for the grouping. The result shows that eighty (80) or $53.0 \%$ of the CHWs were in the group $<36$ years while seventy-two (72) or $47 \%$ of the CHWs were in the group of $>36$ years old.

Education was also analyzed based on the CHWs' educational level ranging from primary to secondary and post- secondary education. Eighteen (18) of the CHWs who had primary education represents $12.0 \%$ of the total number of $152 \mathrm{CHWs}$ that participated in the project. One hundred and thirteen (113) of the CHWs or $74.0 \%$ attained secondary school while twenty-one (21) or $14.0 \%$ of the CHWs attained postsecondary school. Table 1 below provides information about the educational level of the study CHWs.

Occupation was analyzed based on the CHWs' principal work or business ranging from unskilled to skilled. Those trained on the work or business that they do such as fashion designers, health care workers, retired teachers, and soldiers were classified as skilled. While those that are not trained in the work or business that they are involved in are classified as unskilled; and these include petty traders, subsistence farmers, housewives etc. Ninety-six (96) or $63.0 \%$ of the CHWs were unskilled while 56 of the CHWs or $37.0 \%$ of the CHWs were skilled. Most of the CHWs were unskilled $n=96$. Table 1 below provides information about the occupation of the study CHWs.

On the measurement of quality improvement of performance standards disaggregated by rounds. The result shows that during Round 1 CHWs achieved an average of $19(52.2 \%)$ performance standards. This rose to $25(67.5 \%)$ in Round 2; $28(75.6 \%)$ in 3 and $30(81.1 \%)$ in round 4 and $(\mathrm{p}=0.00)$. Therefore, the number of CHWs that scored $>=80$ increased from 79 in round-1 to 124 in round-4. Some of the performance criteria that needed most improvement included reinforcement on checking RDT expiry date, entering results on records, and safe disposing of sharps.

Efforts to improve access to quality case management of febrile illness in Nigeria included the engagement of CDDs to use Rapid Diagnostic tests as a component of home management of malaria (iCCM) and dispense ACTs when the RDTs result is positive and manage pneumonia and diarrhea when negative. It also included effort to monitor and measure the performance of CDDs in providing quality management of febrile illnesses through regular assessments. Four rounds of assessments were conducted among the same cohorts of CHWs, and effects of the measurement recorded and provided in figure- 2 below:

The effect of the regular assessments of the CHWs performance was more on illness management which rose from $45.5 \%$ in round- 1 to $89.1 \%$ in round- 4 . This was followed by history taking and examination increasing from $46.2 \%$ in round- 1 to $80.1 \%$ in round -4 . Conducting RDT for malaria showed slow but continuous increase from $61.5 \%$ in round- 1 to $69.2 \%$ in round- 2 and $76.9 \%$ in round-3 but decreased in round-4 to $72.7 \%$. Performance criteria that needed most improvement included checking signs and symptoms to distinguish among the illnesses in the history taking \& examination thematic area. While CHWs need reinforcement on checking RDT expiry dates, entering RDT results on records and safe disposal of sharps in the Conducting RDT for malaria thematic area; On illness management thematic area CHWs require improvement on counseling for illness preventive measures i.e. reinforcing messages on the use of Long Lasting Insecticide Treated Nets (LLINs) among children under-five years of age and pregnant women; and use of Sulfadoxine-pyrimethamine in prevention of malaria during pregnancy. At the end of each round of assessment, feedback was provided to the CHWs to improve their services before the subsequent round. Feedback provided in between rounds has the effects of helping CHWs improve their performance; and plan for subsequent assessments and feedback sessions as can be seen in figure- 2 . 
Texila International Journal of Public Health

Volume 6, Issue 2, Jun 2018

\section{Discussions}

Discussions centered essentially on the measurement of quality improvement of performance standards. The effects of regular monitoring and measurement of CHWs performance, and how this may have strengthened malaria service delivery was measured. The results of the regular monitoring and measurement of performance standards showed that performance was increasing over time. It was also observed that the CHWs remain the same cohorts, none of them dropped. Though this study did not investigate the reason for that.

The use of one page assessment tool to periodically (two to three months apart) assess and observe CHWs while providing services and using the results to provide feedback in-between rounds of assessments strengthened service delivery by the CHWs during the subsequent round. The feedback mechanism provides the platform for addressing the barriers that limits the CHWs extent of achieving performance standards for improved service delivery. The essence is that the high performing CHWs are engaged to ensure that they continue with quality service delivery while those with areas to improve upon are mentored. So, that promoting interactions between mentors and mentees may improve CHWs achievement of performance standard for quality of iCCM services delivery; thereby decreases high mortality and morbidity due to malaria, pneumonia and diarrhea.

The CHWs achieved performance was observed in each of the three thematic areas - namely history taking and examination; conducting RDTs and illness management. The tools identified sections in the thematic areas that needed more work to improve over time. Example, the sections needed most improvement included checking signs and symptoms to distinguish among the illnesses. CHWs demonstrate eagerness and zeal in the performance of their duties and appear to neglect to check for signs and symptoms to distinguish illness as well as take note of the expiry date of the rapid diagnostic test kits. This is largely due to myths and misconception that every fever is caused by malaria; leading to first treating malaria before investigating other febrile illness only when the treatment of malaria fails. So, the introduction of rapid diagnostic test kits remains innovative in distinguishing malaria from other febrile illness that causes fever.

Also, CHWs needed reinforcement on checking RDT expiry date, entering results on record books, safe disposing of sharps, and counseling on preventive measures. In this anxiety to treat, it was also not unusual to forget checking for expiry date of the RDT first prior to conducting the test; and re-enforcement on preventive measures to maintain good health and fitness. Also, CHWs were under performing in recording RDTs and treatment results. It has been observed that poor documentation of data will limit the ability to improve quality services. This is because if data is not recorded, it cannot be measured and, when it cannot be measured obviously it cannot be improved (Kinney MV., Kerber KJ., Black RE. et al, 2010). This current work recorded improvement in entering results of RDTs in rounds two and three but dropped in round four. Interestingly, the results can be used to plan for additional quarterly assessments and feedback sessions that will strengthen service delivery and improve performance across the thematic areas.

The measurement of quality improvement of performance standards among CHWs and using the results to provide feedback will lead to an increased compliance and adherence to performance standards. Feedback provided in between rounds has the effects of helping CHWs improve their performance; and plan for subsequent assessments and feedback sessions. These findings collaborate the result of an earlier work in Nigeria that monitored and measured quality improvement performance standards among health workers in 23 health facilities in Bauchi State, Nigeria. Results showed that quality of maternal and newborn health services improved in-between rounds and with each assessment (Kabo I., Otolorin EO., Williams E., et al, 2016). The findings also validates the report of another work in Ghana where the monitoring and measurement of provider performance among community-based health services in 2011 - 2014 across 61 community health programs led to over $30 \%$ achievement of performance standards (Necochea E., Tripathi V., and MI Kim Y., et al 2015). CHWs have the potentials to deliver quality management of febrile illness in the current efforts to reduce annual maternal and child deaths while contributing to the achievement of target number 3 of the Sustainable Development Goals (SDGs). In conclusions, CHWs supervisors and 
DOI: $10.21522 / \mathrm{TIJPH} .2013 .06 .02 . A r t 010$

ISSN: $2520-3134$

program managers can use this tool and approach to enhance the quality of services provided by the CHWs and improve their performance through trainings and supervision.

\section{Reference}

[1]. Alam K., Tasneem S., Oliveras E. (2012). Retention of female volunteer community health workers in Dhaka urban slums: a case-control study. Health Policy Plan 27(6): 477-86. Doi: 1093/heapol/czr059.

[2]. Adrienne Kols, Young-Mi Kim, Eva Bazant, Edgar Necochea, Joseph Banda and Stacie Stender, (2015). A Standards-based approach to quality improvement for HIV services at Zambia Defence force facilities: results and lessons learned. AIDS 29(Suppl 2): S145 - S153.

[3]. Ariba AJ., Thanni LO., Adebayo EO. (2007). Patients' perception of quality of emergency care in a Nigerian teaching hospital: The influence of patient-provider interactions. Nigeria Postgraduate. Medical Journal 14(4):296301.

[4]. Bagonza J., Kibira PS., Rutebemberwa E., (2014). Performance of health workers managing malaria, pneumonia and diarrhea under community case management program in central Uganda: a cross sectional study. Malaria Journal doi: 10.1186/1475-2875-13-367.

[5]. Edgar Necochea, Vandana Tripathi, Young MI Kim, Nabeal Akran, Yoland Hyjazi, Maria da Luz Vaz, Emmanuel Otolorin, Tsigue Pleah, Tambudzai Rashidi, Dustan Bishanga (2015). Implementation of Standard-Based Management and Recognition approach to quality improvement in maternal, newborn, and child health programs in low-resource countries. International Journal of Gynecology and Obstetrics 130 S17-S24.

[6]. Hamer DH., Brooks ET., Semrau K., Pilingana P., MacLeod WB., Siazeele K., Sabin LL., Thea DM., YeboahAntwi (2012). Quality and safety of integrated community case management of malaria using rapid diagnostic tests and pneumonia by community health workers. Pathog Glob Health. 106(1): 32-39. doi: 10.1179/1364859411Y.0000000042.

[7]. Harvey S.A, Jennings L, Chinyama M, Masaninga F, Mulholland K, Bell DR, (2008). Improving community health worker use of malaria rapid diagnostic tests in Zambia: package instructions, job aid and job aid-plus-training. Malar J 7: 160.

[8]. Ibrahim Kabo, Emmanuel Otolorin, Emma Williams, Nosa Orobaton, Hannatu Abdullahi, Habib Sadauki, Masduk Abdulkarim, Dele Abegunde. (2016). monitoring maternal and newborn health outcomes in Bauchi State, Nigeria: an evaluation of a standards-based quality improvement intervention. International Journal for Quality in Health Care 28(5), 566-572.

[9]. Kinney MV., Kerber KJ., Black RE, Cohen B., Nkrumah F., Coovadia H., Nampala PM., Lawn JE. (2010) on behalf of the Science in Action: Saving the lives of Africa's mothers, newborns, and children working group. PLoS Med. https://doi.org/10.1371/journal.pmed. 1000294.

[10]. Lopes SC., Cabral A., de Sousa B., (2014). Community health workers: to train or to restrain? A longitudinal survey to assess the impact of training community health workers in the Bolama Region, Guinea-Bissau. Hum Resour Health. 12:8 doi: 10.1186/1478-4491-12-8.

[11]. National Population Commission (NPC), Malaria indicator survey (MIS), 2015.

[12]. Perry HB, Zulliger R., Rogers MM. (2014). Community health workers in low-, middle-, and high-income countries: an overview of their history, recent evolution, and recent effectiveness Annu Rev Public Health. 2014; 35:399-421. doi: 10.1146/annurev-publhealth-032013-182354.

[13]. Young MI Kim, Maureen Chilila, Hildah Shasulwe, Joseph Banda, Webby kanjipite, Supriya Sarkar, Eva Bazant, Cyndi Hiner, Maya Tholandi, Stephanie Reinhardt, Joyce Chongo Mulilo, and Adrienne Kols. (2013). Evaluation of quality improvement intervention of HIV (PMTCT) at Zambia defence force facilities. BMC Health Services Research 13:345.

[14]. World Health Organization - World Malaria Report, 2017. 
Table 1. Socio demographic characteristics of the CHWs

\begin{tabular}{|c|c|c|c|}
\hline & \# of CHWs & $\%$ of CHWs & P-value \\
\hline No. of Participants & $(\mathrm{N}=152)$ & $(\%=100)$ & \\
\hline \multicolumn{4}{|l|}{ Sex } \\
\hline Male & 32 & 21.0 & \\
\hline Female & 120 & 79.0 & \\
\hline \multicolumn{4}{|l|}{ Age } \\
\hline Mean age & $36.8 \pm 8.7$ & & \\
\hline Male mean age & $37.6 \pm 4.3$ & & 0.00 \\
\hline Female Mean age & $36.6 \pm 9.5$ & & \\
\hline$<=36$ & 80 & 53.0 & \\
\hline$>36$ & 72 & 47.0 & \\
\hline \multicolumn{4}{|l|}{ Educational Level } \\
\hline & & & \\
\hline Primary & 18 & 12.0 & \\
\hline Secondary & 113 & 74.0 & \\
\hline Post-secondary & 21 & 14.0 & \\
\hline \multicolumn{4}{|l|}{ Occupation } \\
\hline Unskilled & 96 & 63.0 & \\
\hline Skilled & 56 & 37.0 & \\
\hline
\end{tabular}

Table 2. Measurement of quality improvement standards disaggregated by rounds of assessments and performance score

\begin{tabular}{|l|l|l|l|}
\hline $\begin{array}{l}\text { Total \# of } \\
\text { performance score } \\
=37\end{array}$ & $\begin{array}{l}\text { \# performance } \\
\text { criteria \% } \\
\text { Scored }\end{array}$ & \\
\hline Round-1 & 19 & 52.2 & P-value \\
\hline Round-2 & 25 & 67.5 & \\
\hline Round-3 & 28 & 75.6 & \\
\hline & 30 & 81.1 & 0.00 \\
\hline
\end{tabular}


DOI: $10.21522 /$ TIJPH.2013.06.02.Art010

ISSN: $2520-3134$

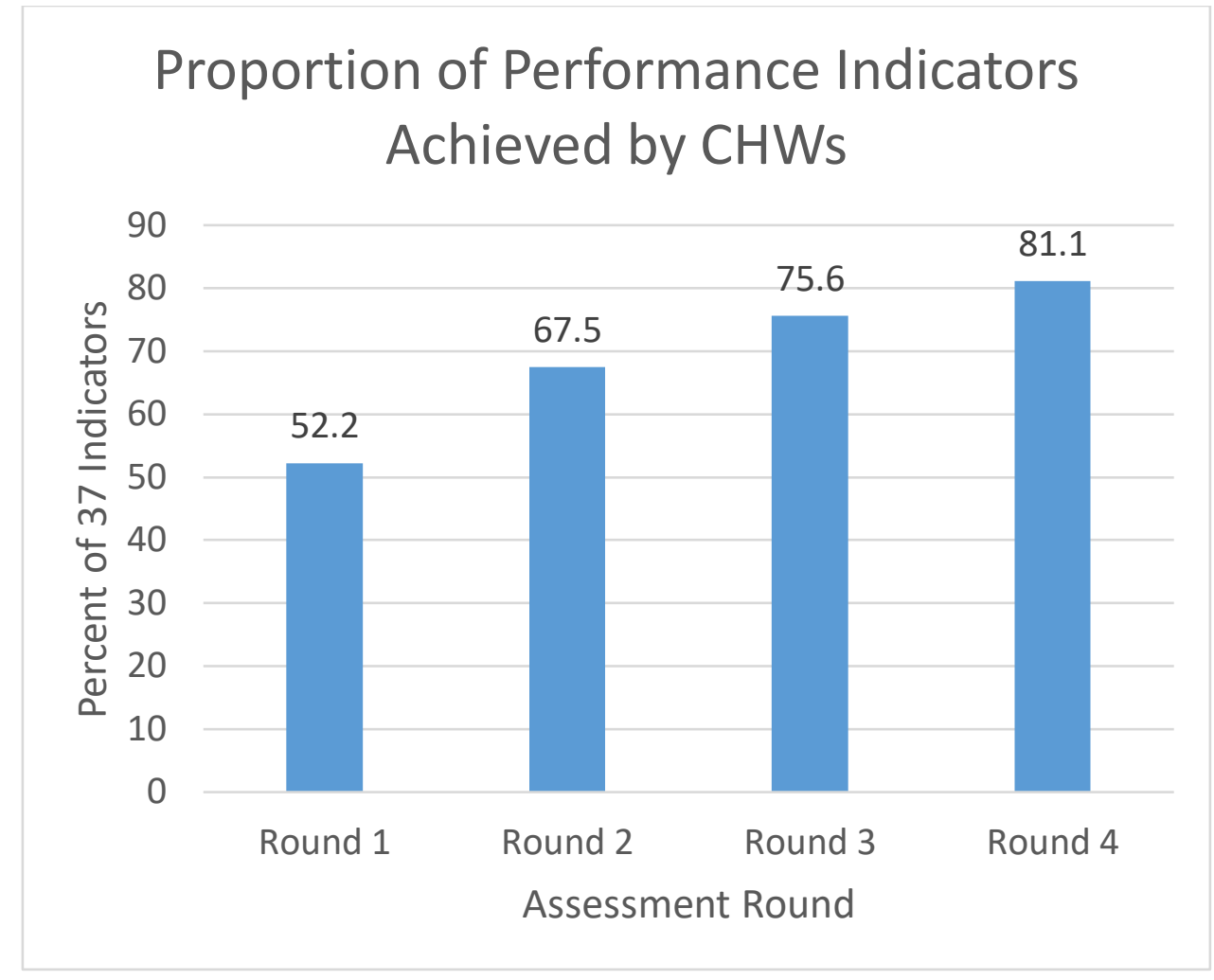

Figure 1. Proportion of performance indicators achieved by community health workers

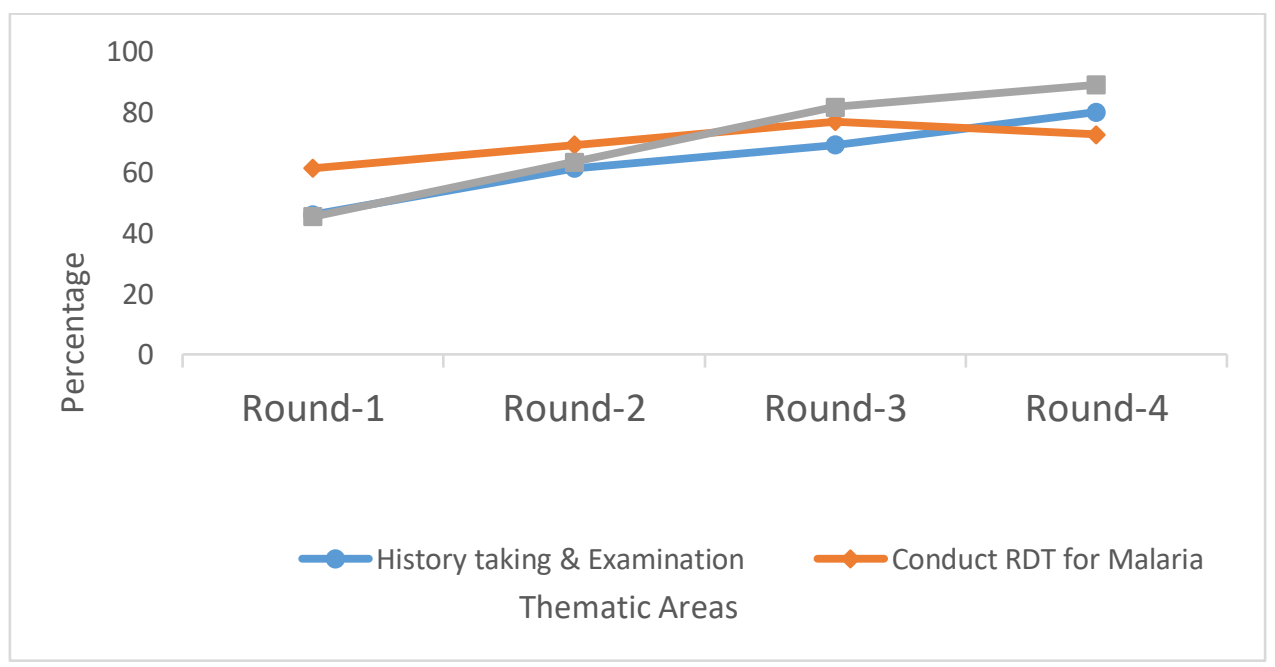

Figure 2. Community health workers performance by treatment sections 